\title{
Stress in mothers of young children with eczema
}

\author{
Jamie Faught, Cynthia Bierl, Belinda Barton, Andrew Kemp
}

Arch Dis Child 2007;92:683-686. doi: 10.1136/adc.2006.112268

See end of article for authors' affiliations

......................

Correspondence to:

Professor Andrew Kemp,

Department of Allergy,

Immunology and Infectious

Diseases, The Children's

Hospital at Westmead,

Locked Bag 4001,

Westmead, Sydney,

New South Wales,

2145 Australia;

andrewk5@chw.edu.au

Accepted 22 March 2007

Published Online First

5 April 2007

......................

\begin{abstract}
Objective: To assess parental stress levels of mothers of children less than 6 years old with eczema and compare these levels with those reported for other chronic childhood illnesses.

Methods: Mothers were recruited from hospital-based out-patient clinics $(55 \%)$ or while their child was an inpatient (45\%) for management of eczema. Maternal stress was measured utilising the Parenting Stress IndexLong Form (PSI) in 33 mothers. The severity of the eczema at the time of interview was documented by the Eczema Area and Severity Index (EASI) score and the Investigators' Global Assessment (IGA) score.

Results: The children with eczema had a mean age of 2.8 years. Mothers of children aged 5 years or less with eczema exhibited significantly higher total stress scores (mean PSI 259.6, 95\% Cl 244.9 to 274.3 ) as compared to mothers of normal children (PSI 222.8, 95\% Cl 221.4 to 224.2) and children with other chronic disorders such as insulin-dependent diabetes (PSI 218.1, 95\% Cl 204.7 to 231.6 ) and profound deafness (PSI $221.7,95 \% \mathrm{Cl} 206.4$ to 237.0$)$. Stress scores in the parental domain $(138.2,95 \% \mathrm{Cl} 128.9$ to 147.6$)$ did not differ significantly from the scores of parents of children with severe disabilities such as those requiring home enteral feeding $(135.2,95 \% \mathrm{Cl} 129.3$ to 141.1$)$ and those with Rett syndrome $(132.8,95 \% \mathrm{Cl} 125.0$ to 140.6).

Conclusions: Moderate to severe childhood eczema should be regarded as a significant illness in which maternal stress is equivalent to that associated with the care of children with severe developmental and physical problems.
\end{abstract}

A topic eczema is a common childhood disorder with a prevalence of $10-16 \%$ in westernised countries. ${ }^{1}$ Caring for a child with moderate to severe eczema involves a rigorous skin treatment regime, adjustments to family lifestyle, and financial and social costs, ${ }^{2-6}$ which all can place substantial demands on the caregivers. Mothers are usually the primary caregivers and carry the major burden in caring for a child with a chronic condition. ${ }^{7}$ Stress will arise if mothers perceive that they cannot adequately cope with these burdens. ${ }^{8}$

There is little literature documenting the stress experienced by mothers who care for a child with eczema. Most studies have examined the impact of atopic dermatitis on the child, family functioning and quality of life, with a few studies examining the parent-child relationship. Studies that have reported on the experiences of mothers indicate that they describe themselves as more depressive, hopeless and anxiously overprotective. ${ }^{9}$ They report feeling stressed about their parenting skills, being less efficient in disciplining their child, and less likely to feel socially supported. ${ }^{10}$

Studies on the family impact of atopic dermatitis have found that the chronicity of the disease, recurrent flare-ups, treatments, costs, and influences on social, career and personal parental realms can significantly compromise family functioning. ${ }^{21-13}$ Families of children with moderate or severe eczema report significantly greater negative impact on family functioning as measured by financial, social and personal domains than that reported by families of children with insulin-dependent diabetes (IDDM). ${ }^{6}$ The quality of life of families of children with eczema is significantly poorer than that of families with healthy children. ${ }^{11}{ }^{13}$ A higher family impact and poorer quality of life are associated with increased eczema severity and more specifically with the parent-caregiver's perception of severity. ${ }^{2}{ }^{13}$

Child characteristics can also contribute to parental stress. For children with eczema, mothers report significantly more behavioural problems, excessive levels of dependency/clinginess and fearfulness, and more sleep difficulties than reported by mothers of children without eczema. ${ }^{10}$ Parents describe their child with eczema as often being distressed, less frequently displaying positive behaviours, naughty and irritable. ${ }^{49}$ Children with eczema often have difficulty sleeping, scratch and itch, and waken during the night. Parents have to manage these symptoms and consequently can lose up to $\mathrm{l}-2 \mathrm{~h}$ of sleep per night, which can lead to decreased coping skills at work and at home. ${ }^{514}$ Mothers of children with eczema who experience a greater disruption to sleep have significantly higher levels of maternal anxiety and depression. ${ }^{5}$

The aims of this study were to: (i) measure the stress levels of mothers of young children with eczema; (ii) to examine the relationship between severity of eczema and maternal stress; and (iii) to compare the stress levels with those associated with other chronic childhood illnesses.

\section{METHODS \\ Subjects}

Thirty eight eligible mothers of children with eczema aged between 1 month and 5 years old were approached to participate. One declined to participate because of time constraints, three mothers did not return the questionnaire, and one was considered to be a defensive responder on the questionnaire, indicating that her answers to questions may not be reliable, and thus was excluded from the analysis. The families were under the care of a paediatric allergist/immunologist (ASK) and/or paediatric dermatologist (MR) in a paediatric hospital-based out-patient allergy or dermatology clinic, or the child was hospitalised for the care of atopic dermatitis. Mothers who were not the child's primary carer and/or not fluent in the English language were excluded. Children with any other significant medical or developmental problems were excluded. Children with allergies (as reported by mothers) and/or asthma were not excluded as these conditions

Abbreviations: EASI, Eczema Area and Severity Index; IDDM, insulindependent diabetes; IGA, Investigators' Global Assessment; PSI, Parenting Stress Index-Long Form; SES, socio-economic status 
frequently coexist. Twenty five mothers were born in Australia and eight elsewhere (East Timor, Hong Kong, Italy, Japan, New Zealand, Philippines, United Kingdom and the USA). Mothers were provided with a study information sheet and signed consent was obtained. The subjects were recruited from July 2004 to May 2005. The Ethics Committee of The Children's Hospital at Westmead approved the study.

\section{Measures}

The amount of stress in the parent-child system was measured using the Parenting Stress Index-Long Form (PSI). ${ }^{8}$ This is a 120 -item questionnaire that measures the sources of stress in the parent-child relationship. The first 101 questions (fivepoint Likert scale format) focus on the sources of stress associated with the child and the parent characteristics, and the last 19 items (yes/no format) assess the level of circumstantial/ demographic life stress. The PSI provides scores for the parent domain and the child domain, to provide a composite total stress score. The parent domain consists of seven subscales: competence, isolation, health, role restriction, depression and spousal relationship. The child domain measures the behavioural and temperamental qualities of the child that make it difficult for parents to fulfil their parental role. It is composed of six subscales: distractibility/hyperactivity, adaptability, reinforces parent, demandingness, mood and acceptability. Higher scores are reflective of higher stress levels. PSI scores greater than the 85 th percentile are in the clinical range, that is, at a level where professional consultation for the stress is indicated. ${ }^{8}$ Mothers completed the PSI while waiting for their child's appointment or at the ward. Due to time constraints, a few mothers completed the PSI at home and returned it by prepaid mail.

At the same visit that the PSI was administered, the severity of eczema was measured by the Eczema Area and Severity Index (EASI $)^{15}$ as determined by one investigator (ASK) and the Investigators' Global Assessment (IGA), which measures the overall severity of eczema using a six-point scale ${ }^{16}$ administered by one dermatologist or allergist/immunologist (ASK). An EASI score of 5 corresponds to mild disease, 12 to moderate disease, 20 to severe disease and 30 to very severe disease. ${ }^{17}$ IGA scores were: 1 almost clear, 2 mild, 3 moderate, 4 severe and 5 very severe. ${ }^{16}$ Socio-economic status (SES) was determined by highest parental occupation using the Australian National University (ANU4) scale (range 0-100), with higher codes assigned to higher paying occupations.

\section{Statistical analysis}

PSI scores were expressed as mean and 95\% CI. Correlations (Pearson's or Spearman's r) were calculated between EASI, PSI and IGA scores. Total stress scores were correlated (Spearman's r) with SES and age of child and $t$ tests were used to

Table 1 Demographic details of the mothers and children

\begin{tabular}{ll}
\hline & $\mathbf{n}(\%)$ \\
\hline Mothers ( $\mathrm{n}=33$ ) & $31(94)$ \\
Marital status (married/partner) & $19(58)$ \\
Mother works outside home & $30(97)$ \\
Partner works outside home & $20(61)$ \\
Have other children & $8(40)^{*}$ \\
Other child with eczema & $18(55)$ \\
Children ( $\mathrm{n}=33$ ) & $14(42)$ \\
Gender: female & $29(88)$ \\
Asthma & \\
Allergies &
\end{tabular}

${ }^{*}$ Calculated using mothers with more than one child $(n=20)$. compare maternal stress levels in children with and without asthma. Mann Whitney was used to compare severity scores by place of recruitment. Level of significance was set at 0.05 and all tests were two-tailed. Data were analysed using Statistical Package for the Social Sciences (SPSS v 12.0.1; SPSS, Chicago, IL).

\section{RESULTS}

The final sample consisted of 33 mothers. Eighteen (55\%) mothers were recruited from out-patient clinics and $15(45 \%)$ from in-patient wards. The mean age of the mothers was 33.8 (SD 5.5) years and the children 2.8 (SD 1.6) years. The mean time since eczema was diagnosed was 2.3 (SD 1.5) years. All children were currently using topical steroid treatments. Median SES score was 63.2 (IQ 38.5) indicating that overall families had slightly higher than average SES and tended to be employed in senior management, public sectors and large organisations. Additional details concerning the mothers and children are shown in table 1.

Mean PSI scores and 95\% CI for mothers are compared to the normative sample and other chronic diseases in table 2. The diabetes, ${ }^{18}$ profound deafness ${ }^{19}$ and cystic fibrosis ${ }^{20}$ data were based on children with a mean age $<5$ years, diabetes 4.6 years, deafness 4.3 years and cystic fibrosis 1.1 years. Children with enteral feeding ( 5.6 years $)^{21}$ and Rett syndrome $(9.4 \text { years })^{22}$ had a mean age $>5$ years. Mothers of children with eczema reported significantly higher levels of stress, as indicated by total stress scores when compared to the normative sample of mothers, parents of children with IDDM and profound deafness (table 2). Mean parental domain scores were significantly higher than the normative sample and children with IDDM, but not significantly different to parents of children with Rett syndrome or requiring home enteral feeding (table 2). The total stress scores of mothers of children with eczema and asthma (258.7) was not significantly different $(\mathrm{p}=0.87)$ compared to mothers of children with eczema alone (260.7).

We examined the proportion of mothers with scores where professional help is recommended (clinical range). For total stress, $46 \%$ of the mothers scored in the clinical range $(>260)$. Of the child domains, $67 \%$ of mothers were in the clinical range $(>22)$ for demandingness, with adaptability the next highest where $55 \%$ of mothers were in the clinical range $(>30)$. Of the parent domains, $52 \%$ of mothers were in the clinical range $(>22)$ for spouse, with competence the next highest with $46 \%$ of mothers in the clinical range $(>35)$.

The overall mean EASI score was 12.3 (SD 8.1) with a range of 0.6-35.7. The EASI score of the hospital in-patients was higher but not significantly so than those in the out-patient clinics (median (IQ) $=15.2 \quad(10.1)$ and median $(\mathrm{IQ})=8.5$ (13.3), respectively; $p=0.10)$. The IGA scores of in-patients and out-patients were the same (median (IQ) $=3(1.00))$. The frequency of the eczema severity scores were (i) IGA: three cases almost clear, eight cases mild, 15 cases moderate, seven cases severe; and (ii) EASI score: 14 cases 0-9, 14 cases 10-19, four cases 20-29 and one case $>30$.

There was a significant positive correlation between EASI and IGA scores (table 3). There were significant positive correlations between EASI, child domain, parent domain and total stress indicating that higher levels of stress were associated with more severe eczema (table 3). There was also a significant positive correlation between demandingness and EASI scores $(\mathrm{r}=0.52, \mathrm{p}=0.002)$. There was a weaker, but still significant correlation with the IGA score and the parent domain and total stress scores (table 3 ). Total stress scores did not significantly correlate with age of the child $(r=0.25$, $p=0.17)$ or SES $(r=-0.23, p=0.19)$. 
Table 2 Comparison between PSI scores for mothers of children with eczema, the normative sample and previous studies of children with other chronic childhood illnesses

\begin{tabular}{|c|c|c|c|c|c|c|c|}
\hline $\begin{array}{l}\text { PSI } \\
\text { domains }\end{array}$ & $\begin{array}{l}\text { Our study, } \\
n=33\end{array}$ & $\begin{array}{l}\text { Normative, } \\
n=2633^{*}\end{array}$ & $\begin{array}{l}\text { Diabetes, } \\
n=40 \dagger\end{array}$ & $\begin{array}{l}\text { Cystic fibrosis, } \\
n=36 \ddagger\end{array}$ & $\begin{array}{l}\text { Rett syndrome, } \\
n=29 \S\end{array}$ & $\begin{array}{l}\text { Deafness, } \\
n=239\end{array}$ & $\begin{array}{l}\text { Enteral feeding, } \\
n=64^{* *}\end{array}$ \\
\hline Child domain & $\begin{array}{l}121.5 \\
(114.3-128.7)\end{array}$ & $\begin{array}{l}99.7 \\
(99.0-100.4)\end{array}$ & $\begin{array}{l}100.9 \\
(94.1-107.7)\end{array}$ & $\begin{array}{l}109.4 \\
(104.3-114.5)\end{array}$ & $\begin{array}{l}131.6 \\
(125.6-137.6)\end{array}$ & $\begin{array}{l}105.9 \\
(98.8-113.0)\end{array}$ & $\begin{array}{l}116.2 \\
(111.3-121.2)\end{array}$ \\
\hline Parent domain & $\begin{array}{l}138.2 \\
(128.9-147.6)\end{array}$ & $\begin{array}{l}123.1 \\
(122.2-124.0)\end{array}$ & $\begin{array}{l}117.2 \\
(109.7-124.7)\end{array}$ & & $\begin{array}{l}132.8 \\
(125.0-140.6)\end{array}$ & $\begin{array}{l}125.4 \\
(114.5-136.3)\end{array}$ & $\begin{array}{l}135.2 \\
(129.3-141.1)\end{array}$ \\
\hline Total stress & $\begin{array}{l}259.6 \\
(244.9-274.3)\end{array}$ & $\begin{array}{l}222.8 \\
(221.4-224.2)\end{array}$ & $\begin{array}{l}218.1 \\
(204.7-231.6)\end{array}$ & & & $\begin{array}{l}221.7 \\
(206.4-237.0)\end{array}$ & $\begin{array}{l}251.4 \\
(242.1-260.7)\end{array}$ \\
\hline
\end{tabular}

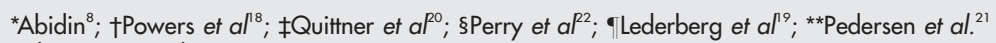

Values expressed as mean $(95 \% \mathrm{Cl})$.

\section{DISCUSSION}

This is the first study utilising a validated measure of stress to report on the magnitude of stress experienced by mothers of young children ( 5 years old or less) with eczema and to correlate the stress with the severity of the eczema. The results of this study indicate that the mothers of children attending hospital-based clinics experience significant stress, with $46 \%$ having a total stress score stress at a level where it is considered professional consultation is indicated. There was a significant association between maternal stress and severity of eczema, with mothers of children with more severe eczema reporting higher levels of stress. Approximately $20 \%$ of the variance in maternal stress scores could be predicted from the variability in eczema severity. In a recent study, Walker et al utilised the PSI to examine parental stress in asthma and eczema in a group of older children (712 years old). ${ }^{23}$ They did not find increased parental stress levels in either asthma or eczema. However, they pointed out that the eczema sample tended towards less pronounced severity and visibility and highlighted the need for studies in more severe cases. Other studies have found that increasing severity of childhood eczema is associated with greater negative impact upon the family and poorer quality of life. ${ }^{11-13} 24$

Child characteristics that contributed to parental stress were the demands and the direct pressures that were placed upon the mother by the child. Most mothers rated their child as being very demanding $(67 \%)$. Children were also rated as being inadaptable $(56 \%)$, that is, unable to adjust to changes in the physical or social environment. Daud et al found that children with eczema were less adaptable and clingier, and that many had a fear of strangers. ${ }^{10}$ It is possible that associated conditions such as asthma or food allergies might also contribute to maternal stress as these conditions commonly coexist with eczema. However, Walker et al found that mothers of children with asthma and eczema did not have significantly different stress levels to those with either condition alone, ${ }^{23}$ and in the present study total stress scores were similar in children with or without asthma.

A lack of emotional and physical support from spouses contributed to elevated maternal stress. We found $27 \%$ of mothers had elevated scores for competency and spouse. Elevated levels of incompetency, coupled with spousal relationship difficulties, suggest a "lack of acceptance and criticism from

Table 3 Correlations between EASI, IGA, child domain, parent domain, and total stress scores

\begin{tabular}{lll}
\hline Scores & EASI & IGA \\
\hline IGA & $0.86^{* *} \dagger$ & - \\
Child domain & $0.44^{*}$ & $0.29 \dagger$ \\
Parent domain & $0.35^{*}$ & $0.38^{*} \dagger$ \\
Total stress & $0.44^{*}$ & $0.29^{*} \dagger$ \\
\hline
\end{tabular}

† Spearman's r.

${ }^{*} p<0.05,{ }^{* *} p<0.01$. the child's other parent". ${ }^{8}$ Previous studies also found that mothers considered the child's eczema had a detrimental effect on their marriage. ${ }^{10}{ }^{13}$ In this study, $46 \%$ of mothers also reported feeling incompetent as a parent concerning the management of their child's behaviour. Other studies have also found that parents feel helplessness and experience disciplinary difficulties with their child. ${ }^{10}$ Mothers with poor spouse support may benefit from educational classes and discussion groups, from which they can learn management techniques and simultaneously experience the emotional support of the group.

Relative to other chronic conditions, mothers of children with eczema reported higher levels of parent domain stress than parents of children with IDDM, ${ }^{18}$ deafness ${ }^{19}$ or recurrent otitis media, ${ }^{25}$ and experienced similar levels to parents of children on home enteral feeding. ${ }^{21}$ This group of children comprises a severely disabled population with multiple disabilities including cerebral palsy, developmental delay, failure to thrive, facial structure defects, congenital heart defects and genetic syndromes, with a mean duration of enteral feeding of 48 months. ${ }^{21}$ Parental stress levels were also of a similar order to those experienced by parents of children with Rett syndrome, a genetic disorder with profound mental retardation, spasticity, seizures, autistic behaviours and sleep disturbance. ${ }^{22}$ These findings indicate that moderate and severe forms of eczema should be regarded as a disorder which induces high levels of stress in the carers rather than as a trivial and temporary cutaneous disease.

Functional family interactions and social networks have been found to favourably influence the course of atopic disease. ${ }^{26}$ Depression and mental distress is common in parents of children with eczema. ${ }^{5} 1012$ In this study, $24 \%$ of mothers had elevated scores for depression. Holm et al recently reported a significant correlation between maternal depression and parental-reported eczema severity. ${ }^{27}$ There is a mutual influence between maternal and child functioning, with maternal depression increasing the risk of adjustment problems in the child. $^{28}$ A recent study found that parental psychological adjustment, the family impact of eczema and a non-supportive family environment were predictive of the child's adjustment difficulties. ${ }^{29}$ Cognitive re-appraisal of stressful events and supportive social networks may lessen the effects of stress. ${ }^{30} 31$

In this study, mothers were recruited from hospital-based clinics and wards where the more severe cases of eczema are more prevalent. Although the median EASI score of the hospital in-patients was higher than that of the out-patients, the difference was not significant; however, it is possible that a significant difference would be observed with larger numbers of subjects. We found a positive correlation between the EASI score and maternal stress. A correlation between a selfadministered form of the EASI score and an eczema family impact scale has also been observed. ${ }^{2}$ Overall, the median EASI and IGA scores corresponded to moderate eczema. It is possible that comparable findings would apply to cases of eczema of similar severity managed in the community as the stress levels 


\section{What is already known on this topic}

- The quality of life of families with children with eczema is poorer than that of families with healthy children.

- The care of a child with moderate or severe eczema has a significantly greater impact on family functioning than the care of children with insulin-dependent diabetes.

\section{What this study adds}

- Stress levels in mothers caring for young children with moderate to severe atopic dermatitis are equivalent to those seen in mothers of children with severe disabilities such as Rett syndrome and children requiring home enteral feeding.

- Maternal stress levels are related to the severity of the atopic dermatitis.

were significantly related to the severity of the dermatitis. Whether or not our results can be extrapolated to cases of similar severity in the community, our findings that almost half $(46 \%)$ of our hospital-recruited cases were associated with maternal stress levels for which professional help is recommended, and that maternal stress is related to the eczema severity, highlight the necessity of clinicians caring for such cases to be aware of this issue. Previous studies found that the impact on family score which assessed financial burden, familial/social impact, personal strain and mastery ${ }^{6}$ and the quality of family life $\mathrm{e}^{11}$ was also related to the severity of the dermatitis. As eczema is a common condition, the impact of this disease, when considered on a community basis, is likely to be substantial. Further research is necessary to identify factors that might alleviate this stress.

\section{ACKNOWLEDGEMENTS}

The contribution of Dr Maureen Rogers to the clinical care and assessment of some IGA scores is gratefully acknowledged.

\section{Authors' affiliations}

Jamie Faught, Cynthia Bierl, Discipline of Paediatrics and Child Health, The Children's Hospital at Westmead Clinical School, University of Sydney, Sydney, Australia

Belinda Barton, Children's Hospital Education Research Institute, The Children's Hospital at Westmead, Sydney, Australia

Andrew Kemp, Department of Allergy, Immunology and Infectious Diseases, The Children's Hospital at Westmead, Sydney, Australia

Competing interests: None.

\section{REFERENCES}

1 Williams H, Robertson C, Stewart A, et al. Worldwide variations in the prevalence of symptoms of atopic eczema in the International Study of Asthma and Allergies in Childhood. J Allergy Clin Immunol 1999;103:125-38.
2 Balkrishnan R, Housman TS, Carroll C, et al. Disease severity and associated family impact in childhood atopic dermatitis. Arch Dis Child 2003:88:423-7.

3 Lapidus CS, Schwarz DF, Honig PJ. Atopic dermatitis in children: who cares? Who pays? J Am Acad Dermatol 1993;28:699-703.

4 Lawson V, Lewis-Jones MS, Finlay AY, et al. The family impact of childhood atopic dermatitis: the Dermatitis Family Impact Questionnaire. Br J Dermatol 1998;138:107-13.

5 Moore K, David TJ, Murray CS, et al. Effect of childhood eczema and asthma on parental sleep and well-being: a prospective comparative study. $\mathrm{Br} J$ Dermatol 2006;154:514-18.

6 Su JC, Kemp AS, Varigos GA, et al. Atopic eczema: its impact on the family and financial cost. Arch Dis Child 1997;76:159-62.

7 Midence K. The effects of chronic illness on children and their families: an overview. Genet Soc Gen Psychol Monogr 1994;120:31 1-26.

8 Abidin R. Parenting Stress Index: professional manual, 3rd edn. Lutz, FL: Psychological Assessment Resources, 1995.

9 Pauli-Pott U, Darui A, Beckmann D. Infants with atopic dermatitis: maternal hopelessness, child-rearing attitudes and perceived infant temperament. Psychother Psychosom 1999;68:39-45.

10 Daud LR, Garralda ME, David TJ. Psychosocial adjustment in preschool children with atopic eczema. Arch Dis Child 1993;69:670-6.

11 Ben Gashir MA, Seed PT, Hay RJ. Are quality of family life and disease severity related in childhood atopic dermatitis? J Eur Acad Dermatol Venereol 2002;16:455-62

12 Warschburger P, Buchholz HT, Petermann F. Psychological adjustment in parents of young children with atopic dermatitis: which factors predict parental quality of life? Br J Dermatol 2004;150:304-11.

13 Aziah MS, Rosnah T, Mardziah A. Childhood atopic dermatitis: a measurement of quality of life and family impact. Med J Malaysia 2002;57:329-39.

14 Reid P, Lewis-Jones MS. Sleep difficulties and their management in preschoolers with atopic eczema. Clin Exp Dermatol 1995;20:38-41.

15 Hanifin JM, Thurston $M$, Omoto $M$, et al. The eczema area and severity index [EASI]: assessment of reliability in atopic dermatitis. EASI Evaluator Group. Exp Dermatol 2001;10:11-18.

16 Wahn U, Bos JD, Goodfield M, et al. Efficacy and safety of pimecrolimus cream in the long-term management of atopic dermatitis in children. Pediatrics 2002;110(1 Pt 1):e2.

17 Barbier N, Paul C, Luger T, et al. Validation of the Eczema Area and Severity Index for atopic dermatitis in a cohort of 1550 patients from the pimecrolimus cream $1 \%$ randomized controlled clinical trials programme. $\mathrm{Br} J$ Dermatol 2004; 150:96-102.

18 Powers SW, Byars KC, Mitchell MJ, et al. Parent report of mealtime behavior and parenting stress in young children with type 1 diabetes and in healthy control subjects. Diabetes Care 2002;25:313-18.

19 Lederberg AR, Golbach T. Parenting stress and social support in hearing mothers of deaf and hearing children: a longitudinal study. J Deaf Stud Deaf Educ 2002;7:330-45.

20 Quittner AL, DiGirolamo AM, Michel M, et al. Parental response to cystic fibrosis: a contextual analysis of the diagnosis phase. J Pediatr Psychol 1992; 17:683-704.

21 Pedersen SD, Parsons HG, Dewey D. Stress levels experienced by the parents of enterally-fed children. Child Care Health Dev 2004;30:507-13.

22 Perry A, Sarlo-McGarvey N, Factor DC. Stress and family functioning in parents of girls with Rett syndrome. J Autism Dev Disord 1992;22:235-48.

23 Walker C, Papadopoulos L, Hussein M. Paediatric eczema and psychosocial morbidity: how does eczema interact with parents' illness beliefs? J Eur Acad Dermatol Venereol 2007;21:63-7.

24 Lewis-Jones MS, Finlay AY, Dykes PJ. The Infants' Dermatitis Quality of Life Index. Br J Dermatol 2001;144:104-10.

25 Forgays DK, Hasazi JE, Wasserman RC. Recurrent otitis media and parenting stress in mothers of two-year-old children. J Dev Behav Pediatr 1992;13:321-5.

26 Gustafsson PA, Kjellman NI, Bjorksten B. Family interaction and a supportive social network as salutogenic factors in childhood atopic illness. Pediatr Allergy Immunol 2002;13:51-7.

27 Holm EA, Esmann S, Jemec GB, Parent gender and assessment of infant life quality. J Eur Acad Dermatol Venereol 2006;20:274-6.

28 Elgar FJ, McGrath PJ, Waschbusch DA, et al. Mutual influences on maternal depression and child adjustment problems. Clin Psychol Rev 2004;24:441-59.

29 Dennis H, Rostill H, Reed J, et al. Factors promoting psychological adjustment to childhood atopic eczema. J Child Health Care 2006;10:126-39.

30 Quittner AL, Glueckauf RL, Jackson DN. Chronic parenting stress: moderating versus mediating effects of social support. J Pers Soc Psychol 1990;59:1266-78.

31 Thoits PA. Stress, coping, and social support processes: where are we? What next? J Health Soc Behav 1995; Spec No:53-79. 